\title{
ACTIVIDAD IN VITRO DE EXTRACTOS ETANÓLICOS DE PROPOLEOS DEL BAJO CAUCA ANTIOQUEÑO SOBRE DOS HONGOS FILAMENTOSOS Y UNO LEVADURIFORME
}

\section{IN VITRO ACTIVITY OF ETHANOLIC EXTRACTS OF PROPOLIS FROM EL BAJO CAUCA ANTIOQUEÑO REGION AGAINST TWO FILAMENTOUS FUNGI AND A YEAST}

\author{
Jesús Viloria-Barragán ${ }^{1}$, Jesús Gil-González ${ }^{2}$, Diego Durango-Restrepo ${ }^{3}$, Juan Marín-Loaiza ${ }^{4}$, Guillermo Correa-Londoño ${ }^{5}$
}

Rev. U.D.C.A Act. \& Div. Cient. 19(2): 333-340, Julio-Diciembre, 2016

\begin{abstract}
${ }^{1}$ M.Sc. en Ciencia y Tecnología de los Alimentos. Universidad Nacional de Colombia, sede Medellín, Facultad de Ciencias Agrarias, Departamento de Ingeniería Agrícola y de Alimentos. Medellín, Colombia, e-mail: jdviloriab@unal.edu.co; ${ }^{2}$ Profesor Asociado. Universidad Nacional de Colombia, sede Medellín, Facultad de Ciencias Agrarias, Departamento de Ingeniería Agrícola y Alimentos. Medellín, Colombia; e-mail: jhgilg@unal.edu.co; ${ }^{3}$ Profesor Asociado. Universidad Nacional de Colombia, sede Medellín, Facultad de Ciencias, Escuela de Química. Medellín, Colombia, e-mail: dldurango@unal.edu.co; ${ }^{4}$ Profesor Asociado. Universidad Nacional de Colombia, sede Bogotá, Facultad de Ciencias, Departamento de Farmacia. Bogotá, Colombia, carrera 30 No. 45-03. Edificio 450, e-mail: jcmarinlo@unal.edu.co; ${ }^{5}$ Profesor Asociado. Universidad Nacional de Colombia, sede Medellín, Facultad de Ciencias Agrarias, Departamento de Agronomía, calle 59A No.63-20, Autopista Norte. Medellín, Colombia, e-mail: gcorrea@unal.edu.co
\end{abstract}

\section{RESUMEN}

El propóleos es una resina colectada por las abejas Apis mellifera de especies botánicas, próximas a los apiarios. Este material resinoso es ampliamente reconocido por sus propiedades antimicrobianas. El objetivo de este trabajo fue evaluar la actividad antifúngica in vitro de los extractos etanólicos de propóleos (EEP), obtenidos a partir de muestras provenientes de apiarios experimentales de investigación, ubicados en la región del Bajo Cauca antioqueño, sobre dos hongos filamentosos, Botryodiplodia theobromae y Fusarium oxysporum y un hongo levaduriforme, Candida albicans. El propóleos fue recolectado por los métodos de malla y de raspado, durante diferentes periodos del año. Mediante la técnica de macrodilución en caldo, se evaluaron seis dosis de EEP, en un rango de156 a $5000 \mu \mathrm{gmL}^{-1}$ y se determinó la concentración que inhibió el 80 y el 50\% del crecimiento del hongo. Los resultados indican que el método de recolección del propóleos presentó diferencias significativas con respecto al crecimiento de $C$. albicans y no para $B$. theobromae y $F$. oxysporum. Las CMlso estuvieron entre 1211 a $4050 \mu \mathrm{gmL}^{-1}, 1567$ a $5016 \mu \mathrm{gmL}^{-1}$ y 1529 a $3568 \mu \mathrm{gmL}^{-1}$ para $B$. theobromae, $F$. oxysporum y $C$. albicans, respectivamente. En conclusión, los extractos evaluados exhibieron un actividad inhibitoria del crecimiento moderada, en comparación con el control y el hongo levaduriforme, $C$. albicans presentó la mayor sensibilidad frente a los EEP analizados. Adicionalmente, el método de recolección del propóleos solo presentó diferencias significativas respecto a la concentración mínima inhibitoria, encontrada para C. albicans.

Palabras clave: B. theobromae, F. oxysporum, C. albicans, Agentes antimicóticos.

\section{SUMMARY}

Propolis is a resin collected by the Apis mellifera bees from botanical species surrounding the apiaries. This resinous material is widely renowned by its antimicrobial properties. The aim of this research was to evaluate the in vitro activity of ethanolic extracts from propolis (EEP), obtained from experimental research apiaries located in the Bajo Cauca antioqueño region, against two filamentous fungi, Botryodiplodia theobromae and Fusarium oxysporum, and a yeast, Candida albicans. Propolis were collected in different periods of the year, by methods of scraping and mesh. Using the macrodilution technique, six doses of EEP ranging from 156 to $5000 \mu \mathrm{gmL}^{-1}$ were evaluated and minimal inhibitory concentration 80 and inhibitory concentration 50 were determined. Results indicated that the harvest method showed a significant effect on C. albicans growth, but not for $B$. theobromae and F. oxysporum. The MICs8o observed were between 1211 to $4050 \mu \mathrm{gmL}^{-1}, 1567$ to $5016 \mu \mathrm{gmL}^{-1}$ and 1529 to $3568 \mu \mathrm{gmL}^{-1}$ for $B$. theobromae, F. oxysporum and C. albicans, respectively. In conclusion, the extracts tested showed a moderate inhibitory growth activity, in comparison with the control; the yeast, $C$. albicans, showed the highest 
sensibility to the EEP evaluated. Additionally, the collection methods only showed significant differences with respect to minimal inhibitory concentration found for $C$. albicans.

Key words: B. theobromae, F. oxysporum, C. albicans, Antifungal agents.

\section{INTRODUCCIÓN}

La contaminación fúngica de los alimentos conlleva no solo a deterioro, alteraciones, podredumbre de tejidos y apariencia poco agradable en las materias primas y productos manufacturados, sino también a la capacidad de algunos hongos de provocar afecciones clínicas, debido a metabolitos, como por ejemplo, las micotoxinas. Entre los hongos filamentosos y fitopatógenos que mayormente afectan los alimentos, se encuentra Fusarium oxysporum, que produce marchitamientos vasculares en cultivos económicamente importantes, como el tomate, el plátano, el garbanzo y el maíz, entre otros (Agrios, 2004; Gómez-Tenorio et al. 2015). Otro hongo fitopatógeno de alta incidencia en cultivos y productos alimenticios es el Botryodiplodia theobromae, moho causante de la pudrición de frutos, como el mango, el banano y el cacao (Twumasi et al. 2014).

Por otra parte, diversos géneros de hongos levaduriformes, como Candida, Cryptococcus y Rhodotorula, son reconocidos por ocasionar alteraciones en los alimentos y las bebidas, causando deterioro y cambios en sus características organolépticas (Orberá-Ratón, 2004). Las levaduras son resistentes a condiciones ambientales extremas, bajo las cuales, otros tipos de microorganismos no se pueden desarrollar (Gouma et al. 2015). Muchas de estas condiciones se ven favorecidas cuando se realizan procesos de conservación en alimentos, tales como la adición de ácidos orgánicos, la concentración con solutos y la evaporación, entre otros; sin embargo, hay alimentos que se ven más afectados por hongos levaduriformes, como es el caso de los jugos, concentrados de frutas, los quesos, las frutas y los vegetales (Orberá-Ratón, 2004; Ceugniez et al. 2015).

Debido al alto grado de residualidad, el uso excesivo de preservantes y de conservantes sintéticos en la industria alimenticia ha generado problemas adversos en la salud del consumidor (Shahmihammadi et al. 2016) y, en otros casos, han propiciado ambientes favorables para el crecimiento de microorganismos alteradores (Orberá-Ratón, 2004). Adicional a estos inconvenientes, las empresas de alimentos se encuentran con leyes cada vez más estrictas y con la necesidad de suplir la gran demanda, por parte de los consumidores, de conservantes y de preservantes, de origen natural. En los últimos años, productos como quitosano, bacteriocinas, aceites esenciales, propóleos, entre otros, han surgido como una alternativa a los métodos de control microbiano tradicionales, debido a las ventajas, como bajos costos, bio- degradabilidad y biocompatibilidad, que presenta el empleo de productos de origen natural (Gyawali \& Ibrahim, 2014; Siripatrawan \& Vitchayakitti, 2016).

El propóleos es un producto natural resinoso, acopiado por las abejas Apis mellifera, a partir de heridas y de exudados de diversas especies botánicas. A este producto de la colmena, se le han atribuido múltiples beneficios y actividades biológicas, tales como antioxidante, antibacteriana, antifúngica, entre otras (Yang et al. 2011; Graikou et al. 2016). Diversos autores consideran que este material resinoso puede ser considerado como una alternativa promisoria para el control de hongos fitopatógenos, que podría ser empleada en el ámbito industrial (Jiao \& Yuan, 2004). Asimismo, un gran número de estudios llevados a cabo contra hongos fitopatógenos de diversos géneros, como Fusarium, Botryodiplodia, Colletotrichum, Aspergillus, Penicillium, Candida y otros, validan la actividad antimicótica que el propóleos presenta (Quiroga et al. 2006; Meneses et al. 2009; Palomino et al. 2010; Freires et al. 2016).

Teniendo en cuenta que los estudios para establecer el efecto antifúngico de propóleos de origen colombiano son incipientes, el presente trabajo de investigación tuvo como objetivo evaluar la actividad de los extractos etanólicos obtenidos, a partir de propóleos recolectados en la región del Bajo Cauca antioqueño, frente a hongos filamentosos y uno levaduriforme.

\section{MATERIALES Y MÉTODOS}

Obtención de las muestras de propóleos. El propóleos crudo, se recolectó en la subregión del Bajo Cauca antioqueño-Colombia. Tres apiarios, ubicados en los municipios de Zaragoza, El Bagre y Caucasia, fueron elegidos al azar y denominados como Llanta Azul (LA), Doña María (DM) y Coco Hondo $(\mathrm{CH})$, respectivamente. Las muestras, se cosecharon por los métodos de raspado $(R)$ y malla $(M)$, durante tres periodos: junio-agosto 2009 (periodo 1), agosto-noviembre 2009 (periodo 2) y marzo-mayo 2010 (periodo 3). El muestreo, se efectuó de forma aleatoria en las diferentes colmenas de los apiarios. En el área de ubicación de los apiarios predominaron las especies vegetales Acacia mangium, Mimosa pigra y matorrales. Un total de 18 muestras fueron almacenadas y conservadas a $-18^{\circ} \mathrm{C}$.

Preparación de los extractos etanólicos de los propóleos (EEP). A $10 \mathrm{~g}$ del propóleos crudo se le adicionaron $300 \mathrm{~mL}$ de etanol acuoso, al $70 \%$ (v/v). La mezcla obtenida fue sometida a ultrasonido durante $3 \mathrm{~h}$; posteriormente, se retiró el material insoluble mediante filtración; el filtrado fue enfriado a $-18^{\circ} \mathrm{C}$ durante $24 \mathrm{~h}$, para precipitar las ceras, que fueron eliminadas por filtración. La solución se evaporó a sequedad bajo presión reducida a $45^{\circ} \mathrm{C}$ y el residuo (EEP) fue almacenado a $-18^{\circ} \mathrm{C}$, hasta su utilización. 
Material biológico. Para la evaluación de la actividad antifúngica in vitro, se utilizaron cepas de los hongos Botryodiplodia theobromae, donado por el Laboratorio de Fitopatología de la Universidad Nacional de Colombia -sede Medellín-; Fusarium oxysporum, proporcionado por la Corporación Colombiana de Investigación Agropecuaria -CORPOICA-, sede Palmira y la cepa de referencia ATCC 102321 de Candida albicans. Las esporas de los hongos filamentosos $B$. theobromae y $F$. oxysporum fueron obtenidas de acuerdo con la metodología descrita por Rivillas-Acevedo \& Soriano-García (2007) y fueron almacenadas a $-20^{\circ} \mathrm{C}$. Para C. albicans, se preparó una concentración de $1 \times 10^{4} \mathrm{UFC} / \mathrm{mL}$, a partir de una concentración conocida de $1 \times 10^{8} \mathrm{UFC} / \mathrm{mL}$, correspondiente al patrón McFarland 0,5.

Evaluación de la actividad antifúngica. Las pruebas de actividad antifúngica, se realizaron en tubos de ensayo, siguiendo la metodología desarrollada por Rivillas-Acevedo \& Soriano-García (2007) y los protocolos de macrodilución M38-A (CLSI, 2002) y M27-A (CLSI, 1997), para hongos filamentosos y levaduras, respectivamente. Un total de seis concentraciones de EEP, en el rango de 156 a $5000 \mu \mathrm{gmL}^{-}$

${ }^{1}$, fueron utilizadas para evaluar la inhibición del crecimiento de los hongos $B$. theobromae, F. oxysporum y C. albicans. $100 \mathrm{mg}$ de EEP disueltos en $250 \mu \mathrm{L}$ de dimetilsulfóxido (DMSO) y 50mg de Tween 80 se diluyeron, hasta $1000 \mu \mathrm{L}$ con agua destilada estéril; a partir de esta disolución, se preparó $400 \mu \mathrm{L}$ de la solución de concentración $5000 \mu \mathrm{gmL}^{-1}$ y mediante dobles diluciones seriadas con agua destilada estéril, se prepararon $200 \mu \mathrm{L}$ de las dosis inferiores, hasta una concentración de $156 \mu \mathrm{gmL}^{-1}$. Seguidamente, en cada tubo' se adicionaron $700 \mu \mathrm{L}$ de caldo Sabouraud y $100 \mu \mathrm{L}$ de inóculo, que contenía $2 \times 10^{4}$ esporas $/ \mathrm{mL}$, para los hongos filamentosos o $1,5 \times 10^{4}$ levaduras $/ \mathrm{mL}$, para el hongo levaduriforme. Como control negativo, se emplearon $200 \mu \mathrm{L}$ de la solución dispersante en $700 \mu \mathrm{L}$ de medio de cultivo y como control positivo timol (Abaquin Ltda.).

Los tratamientos y los controles fueron incubados a $37^{\circ} \mathrm{C}$ durante $24 \mathrm{~h}$, para el hongo levaduriforme y a $25^{\circ} \mathrm{C}$ por $48 \mathrm{~h}$, para los hongos filamentosos (CLSI, 2002). Los bioensayos fueron realizados por triplicado. Las absorbancias fueron leídas en un espectrómetro UV-1800 Shimadzu, a una longitud de onda de $600 \mathrm{~nm}$.

La inhibición del crecimiento microbiano se determinó con la siguiente fórmula:

Inhibición $\left.(\%)=\left(\left(\left(A c_{x}-A c_{y}\right)-\left(A t_{x}-A t_{y}\right)\right) /\left(A c_{x}-A c_{y}\right)\right)\right) x 100$

Dónde: Acx y Atx son las absorbancias del control negativo y el tratamiento, respectivamente, calculadas al final del periodo de crecimiento de los microrganismos y Acy y Aty, absorbancia del control negativo y el tratamiento, medidas a los 30min de iniciada la evaluación. El denominador está determinado por la diferencia de la absorbancia corregida del control negativo menos la absorbancia corregida de los tratamientos. La absorbancia corregida, se definió como la diferencia entre la absorbancia medida al final del periodo de crecimiento de los microorganismos y la absorbancia a los 30 minutos de iniciado el ensayo.

La concentración mínima inhibitoria 80 (CMI80) se definió como la concentración que inhibió el $80 \%$ del crecimiento del hongo y la concentración inhibitoria 50 (Cl50) fue considerada como la dosis responsable del $50 \%$ de la inhibición del hongo. La concentración fue determinada mediante un análisis de regresión lineal, realizada entre los porcentajes de inhibición y la dosis. La curva se interpoló a un porcentaje de inhibición del $50 \%$ y se leyó la concentración correspondiente.

Análisis estadístico. Se evaluó la actividad antifúngica del propóleos sobre tres hongos, a través de la $\mathrm{CMI}_{80}$ y la $\mathrm{Cl}_{50}$. En cada apiario, se evaluaron dos técnicas de recolección de los propóleos: raspado y malla, durante tres periodos. Al considerar que los apiarios y los periodos de recolección son factores aleatorios, únicamente se evaluaron los dos niveles del método de recolección, empleando un diseño de bloques completos al azar, con nueve repeticiones. El Anava, se llevó a cabo siguiendo el procedimiento Mixed del software SAS, versión 9.00. Adicionalmente, se elaboró una representación HJ-Biplot (Galindo, 1986), para las dos variables respuesta, en cada uno de los tres hongos y las 18 observaciones, mediante una rutina elaborada en el software Matlab, versión 7.6.0.32.

\section{RESULTADOS Y DISCUSIÓN}

En la tabla 1, se aprecia que los EEP mostraron una actividad antifúngica moderada frente a los hongos filamentosos y la levadura. Los valores CMl8o más bajos fueron de 1211 y $1567 \mu \mathrm{g} / \mathrm{mL}$, para $B$. theobromae y F.oxysporum, a las $48 \mathrm{~h}$, respectivamente y $1529 \mu \mathrm{g} / \mathrm{mL}$, para $C$. albicans, a las $24 \mathrm{~h}$. Respecto a los métodos de recolección malla y raspado, solo se observó una diferencia significativa en la CMlso para $C$. albicans, valor $\mathrm{P}=0,03$.

Las $\mathrm{CMI}_{80}$ para el fitopatógeno $B$. theobromae variaron en un rango amplio, con valores comprendidos entre 1211 y $4050 \mu \mathrm{gmL}^{-1}$ (Tabla 1). Adicionalmente, se observó que, en promedio, las muestras colectadas por el método de malla presentan valores de $\mathrm{CMI}_{80}$ inferiores a los arrojados por el método de raspado. Los valores de Cl5o para $B$. theobromae oscilaron entre 163 y $1522 \mu \mathrm{gmL}^{-1}$ (Tabla 1 ) y no se detectaron diferencias significativas entre los métodos de recolección, valor $\mathrm{P}=0,15$. 
Tabla 1. Determinación de la concentración mínima inhibitoria 80 y concentración de inhibición 50, para los extractos etanólicos de propóleos, de la región del Bajo Cauca antioqueño.

\begin{tabular}{|c|c|c|c|c|c|c|}
\hline \multirow{3}{*}{ MUESTRA } & \multicolumn{6}{|c|}{ Microorganismos } \\
\hline & \multicolumn{2}{|c|}{ B. theobromae } & \multicolumn{2}{|c|}{ F. oxysporum } & \multicolumn{2}{|c|}{ C. albicans } \\
\hline & $\begin{array}{l}\text { CMI } 80 \\
(\mu \mathrm{g} / \mathrm{mL})\end{array}$ & $\mathrm{CI}_{50}(\mu \mathrm{g} / \mathrm{mL})$ & $\begin{array}{l}\text { CMI } 80 \\
(\mu \mathrm{g} / \mathrm{mL})\end{array}$ & $\mathrm{CI}_{50}(\mu \mathrm{g} / \mathrm{mL})$ & $\begin{array}{l}\text { CMI } 80 \\
(\mu \mathrm{g} / \mathrm{mL})\end{array}$ & $\mathrm{CI} 50(\mu \mathrm{g} / \mathrm{mL})$ \\
\hline LA1R & 3477 & 908 & 5016 & 1872 & 2236 & 657 \\
\hline LA1M & 2302 & 645 & 4581 & 1943 & 3288 & 1502 \\
\hline DM1R & 2427 & 620 & 4000 & 1097 & 1667 & 759 \\
\hline DM1M & 2725 & 780 & 4595 & 1817 & 2673 & 811 \\
\hline $\mathrm{CH} 1 \mathrm{R}$ & 3650 & 1127 & 2654 & 730 & 2459 & 1134 \\
\hline $\mathrm{CH} 1 \mathrm{M}$ & 1364 & 163 & 3459 & 1493 & 1529 & 632 \\
\hline LA2R & 4050 & 1522 & 2880 & 859 & 2659 & 1215 \\
\hline LA2M & 2991 & 810 & 4828 & 1707 & 3467 & 1232 \\
\hline $\mathrm{DM} 2 \mathrm{R}$ & 2239 & 641 & 2418 & 771 & 2884 & 920 \\
\hline DM2M & 2590 & 574 & 4290 & 1007 & 3568 & 1056 \\
\hline $\mathrm{CH} 2 \mathrm{R}$ & 1211 & 182 & 3144 & 621 & 3219 & 1091 \\
\hline $\mathrm{CH} 2 \mathrm{M}$ & 1531 & 634 & 2322 & 931 & 2883 & 895 \\
\hline LA3R & 4043 & 1509 & 2224 & 467 & 2203 & 618 \\
\hline LA3M & 2522 & 477 & 1858 & 470 & 3174 & 748 \\
\hline DM3R & 3788 & 880 & 2125 & 558 & 1884 & 475 \\
\hline DM3M & 2801 & 766 & 3610 & 941 & 3097 & 919 \\
\hline $\mathrm{CH} 3 \mathrm{R}$ & 3377 & 829 & 1567 & 264 & 2431 & 606 \\
\hline $\mathrm{CH} 3 \mathrm{M}$ & 2258 & 556 & 1745 & 285 & 2952 & 498 \\
\hline Control $^{+}$ & 67 & & 105 & & 81 & \\
\hline
\end{tabular}

${ }^{+}$Control positivo Timol (Abaquin, Ltda.). LA, DM y CH corresponde al nombre de los apiarios Llanta Azul, Doña María y Coco Hondo, respectivamente. M y R indican el método de recolección del propóleos (malla o raspado) y la numeración 1, 2 y 3 hace referencia al periodo de recolección.

En los bioensayos realizados con $F$. oxysporum, las CMI80 fluctuaron en valores comprendidos entre 1567 y $5016 \mu \mathrm{gmL}^{-}$ ${ }^{1}$ (Tabla 1). Los propóleos colectados por el método de raspado exhibieron concentraciones de inhibición menores contra F. oxysporum, que las muestras colectadas por el método de malla; no obstante, no se presentaron diferencias estadísticas. Por su parte, los valores de $\mathrm{Cl}_{50}$ para $F$. oxysporum se encontraron en el rango de 264 y $1943 \mu \mathrm{gmL}^{-1}$ (Tabla 1), apreciándose diferencias significativas entre los propóleos colectados por los métodos de malla y raspado, valor $\mathrm{P}=0,009$.
En las evaluaciones de los EEP realizadas sobre la cepa de referencia C. albicans ATCC 102321, se observó que el rango de concentraciones, en donde se inhibía el $80 \%$ de la germinación de la levadura, comprende desde 1529 hasta $3568 \mu \mathrm{gmL}^{-}$ ${ }^{1}$ (Tabla 1). En general, para la evaluación antifúngica sobre $C$. albicans los resultados evidenciaron que los EEP colectados por el método de raspado presentaron valores promedios de CMlso menores, con respecto a los mostrados por los EEP colectados por el método de malla. A su vez, los valores de Cl50 estuvieron en el rango de 475 a $1502 \mu \mathrm{gmL}^{-1}$ (Tabla 1); no obstante, no se presentaron diferencias significativas entre los 
métodos de recolección para los valores de Cl50 reportados para C. albicans, valor $\mathrm{P}=0,43$.

Finalmente, se construyó una representación HJ-Biplot (Figura 1), con el fin de visualizar las relaciones entre las variables evaluadas sobre los tres tipos de hongos y su posible asociación con los métodos de recolección (raspado y malla). No se observó que el método de recolección haya generado diferencias en $\mathrm{CMI}_{80}$ y $\mathrm{Cl}_{50}$ para los hongos filamentosos $B$. theobromae y $F$. oxysporum; esto se colige del hecho de que los métodos de recolección no se separan al ser proyectados sobre cualquiera de los vectores correspondientes a los parámetros evaluados, sobre estos dos hongos. Asimismo, el estrecho ángulo que para cada uno de estos dos hongos forman los vectores correspondientes a los parámetros $\mathrm{CMI}_{80}$ y $\mathrm{Cl}_{50}$ es reflejo de la alta correlación positiva existente, entre tales parámetros. Al evaluar los propóleos sobre el hongo levaduriforme $C$. albicans, los métodos sí adquieren un buen nivel de separación al ser proyectados sobre el vector correspondiente a la CMlso, siendo mayor cuando se utiliza el método de raspado. Para este hongo, la correlación entre parámetros no es tan fuerte.

Los hongos $B$. theobromae y $F$. oxysporum, afectan grandes extensiones de cultivos de importancia económica en Colom- bia, como es el caso de aguacate, mango, maíz, entre otros. Por otra parte, los hongos levaduriformes, particularmente los pertenecientes al género Candida, se pueden encontrar como contaminadores de alimentos procesados. Debido a los problemas que son inherentes a las sustancias conservantes de origen sintético (Pop et al. 2016), actualmente el uso de productos de origen natural se ha convertido en una alternativa para controlar estos microorganismos (Lucera et al. 2012). Dentro de los productos naturales, se registran los propóleos, sustancias resinosas ampliamente reconocidas por sus propiedades antimicrobianas (Bankova et al. 2014). Por otro lado, diversos autores han reportado la actividad antifúngica de los propóleos frente a hongos filamentosos fitopatógenos, como Penicillium spp., Aspergillus spp., Fusarium spp., Botryodiplodia spp. y hongos levaduriformes, especialmente, los del género Candida (Quiroga et al. 2006; Meneses et al. 2009; Agüero et al. 2011; Yang et al. 2011).

En este estudio, se determinó el efecto antifúngico de los EEP de la región del Bajo Cauca antioqueño. La actividad antimicrobiana del propóleos se halla íntimamente relacionada con la composición química (Toreti et al. 2013), la cual, puede variar de acuerdo a su lugar de origen; por ejemplo, los propóleos de origen europeo están compuestos, en la mayoría, por sustancias fenólicas, principalmente flavonoi-

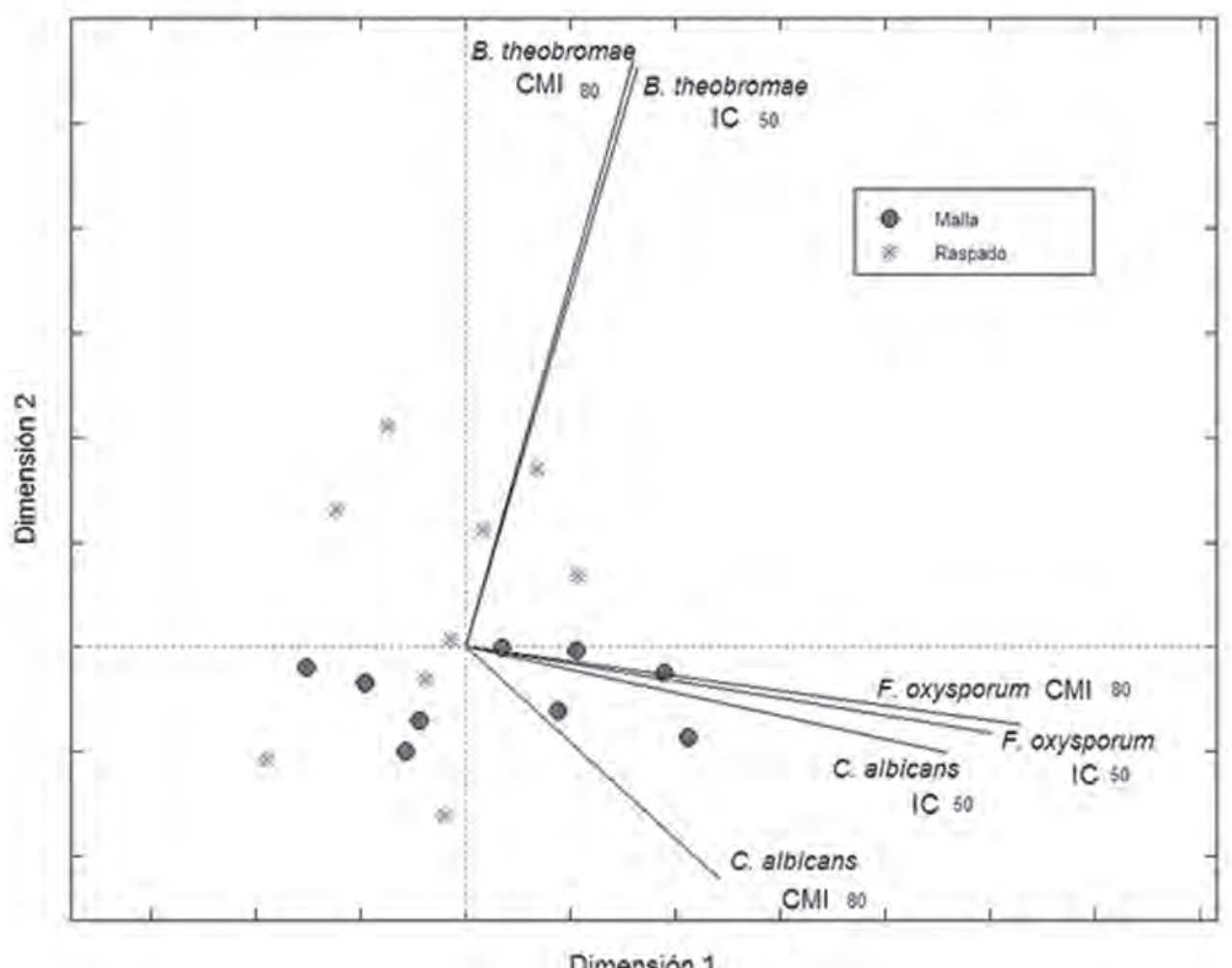

Figura 1. Representación Biplot de la población muestral frente a los patrones CMI80 e IC50. 
des, como galangina y pinocembrina (Salatino et al. 2011). Por su parte, Melliou et al. (2007) estudiaron la actividad antimicrobiana de los constituyentes del propóleos de varias regiones de Grecia, en los cuales, predominaron terpenoides, y mostraron mayor actividad sobre los hongos. Para el caso colombiano, Meneses et al. (2009) y Palomino et al. (2010) caracterizaron propóleos colectados en Antioquia, Colombia y establecieron que la actividad antimicrobiana apreciada frente a los hongos de los géneros Botryodiplodia, Colletotrichum, Aspergillus y Penicillum, puede estar relacionada con el contenido de diterpenos tipo labdano o al sinergismo de éstos con otros componentes. Además, se estima que no es solo una sustancia la responsable de la actividad, sino más bien que es debida al efecto sinérgico de diferentes compuestos (Barbarić et al. 2011). Estudios complementarios de la composición química de los propóleos del Bajo Cauca antioqueño permitirán vislumbrar la relación presente entre la actividad manifestada por los EEP con sus constituyentes.

Por otro lado, no se establecieron resultados concluyentes respecto a la influencia del método de recolección sobre la actividad biológica; solo en el hongo levaduriforme, se presentaron diferencias significativas en la $\mathrm{CMI}_{80}$, respecto al método de recolección. Posiblemente, $C$. albicans es un microorganismo más sensible al EEP que los hongos filamentosos y, por lo tanto, su crecimiento es afectado, aún con sutiles diferencias en la concentración y en la composición del extracto. Papotti et al. (2012) establecieron que la composición y la actividad biológica de los extractos depende del método de recolección del propóleos, debido a que hay variaciones en la concentración de resinas y de bálsamos, que son las fracciones que contienen las sustancias bioactivas, entre las muestras, obtenidas por malla y por raspado.

El hongo $F$. oxysporum fue la especie menos sensible al EEP del Bajo Cauca antioqueño, con valores de $\mathrm{CMI}_{80}$, entre 1567 y $5016 \mu \mathrm{gmL}^{-1}$. Estos resultados son comparables a los presentados por extractos metanólicos de propóleos provenientes de cinco regiones de Turquía, frente a Fusarium oxysporum f. sp. melonis (Özcan et al. 2004), donde la concentración de $2000 \mu \mathrm{gmL}^{-1}$ fue efectiva para la inhibición del hongo, en un 50\%. De igual forma, Özcan (1999) registró porcentajes de inhibición de F. oxysporum superiores al $50 \%$, cuando evaluó extractos acuosos de propóleos colectados en Konya-Turquía, a concentraciones de $4000 \mu \mathrm{gmL}^{-1}$. Los EEP del Bajo Cauca probados a dosis inferiores a $4000 \mu \mathrm{gmL}^{-1}$ inhibieron el $80 \%$ de la germinación de los conidios del fitopatógeno F. oxysporum. Por su parte, Quiroga et al. (2006) hallaron concentraciones mínimas inhibitorias de 349, 310 y $194 \mu \mathrm{gmL}^{-1}$, para Fusarium sp., obtenido de tres aislados de Glycine max; no obstante, los autores utilizaron un extracto etanólico de propóleos parcialmente purificado, que presentaba, como compuestos mayoritarios, pinocembrina y galangina.
Son pocos los reportes relacionados con la actividad antifúngica de EEP frente a $B$. theobromae; sin embargo, autores como Meneses et al. (2009), cuando utilizaron extractos de propóleos procedentes de Antioquia, Colombia, a una concentración de $1000 \mu \mathrm{gmL}^{-1}$, reportaron inhibición en el crecimiento micelial, del $29 \%$.

El potencial antifúngico de EEP frente al hongo levaduriforme C. albicans ha sido estudiado por diversos autores (Agra et al. 2006; Suleman et al. 2015; Freires et al. 2016). La levadura $C$. albicans fue la especie más susceptible al EEP del Bajo Cauca, con valores $\mathrm{CMI}_{80}$, entre 1529 y $3568 \mu \mathrm{gmL}^{-1}$. Stepanović et al. (2003) indicaron que los EEP colectados en diferentes regiones de Serbia mostraron CMI en el rango de 0,31 a 2,5\%; adicionalmente, Abd \& Hegazi (2000) encontraron valores CMI entre 1320 y $3380 \mu \mathrm{gmL}^{-1}$, en EEP, provenientes de Egipto, rango similar al hallado para EEP del Bajo Cauca. Por otra parte, autores como Herrera et al. (2010), han reportado rangos de CMI entre 197 y $441 \mu \mathrm{gmL}^{-1}$, de extractos líquidos comerciales de propóleos chilenos, para la inhibición del hongo levaduriforme $C$. albicans, al emplear la técnica de microdilución.

Este hecho sugiere el potencial de los EEP como alternativa para el tratamiento de enfermedades, de alteraciones y de contaminaciones causadas por hongos filamentosos y levaduras, que atacan frutos, hortalizas, granos, alimentos preparados, entre otros. Igualmente, será de gran relevancia estudios adicionales in vivo, que sustenten estas afirmaciones.

En conclusión, los extractos exhibieron una actividad moderada, en comparación con el control, contra los hongos B. theobromae, F. oxysporum, y C. albicans; además, el microorganismo levaduriforme presentó la mayor sensibilidad frente a los EEP evaluados in vitro. De igual manera, se evidenció que el método de recolección del propóleos solo mostró diferencias significativas respecto a la concentración mínima inhibitoria encontrada para C. albicans.

Agradecimientos: La presente investigación, se realizó gracias a la financiación otorgada por el Ministerio de Agricultura y Desarrollo Rural de Colombia, por la Universidad Nacional de Colombia y por la empresa Promoción de Proyectos Mineros S.A.S., a través del proyecto 003-2008C3783-3453. Conflicto de intereses: Los autores declaramos que no existe conflicto de intereses que ponga en riesgo la validez de los resultados presentados.

\section{BIBLIOGRAFÍA}

1. ABD, E.; HEGAZI, A. 2000. Egyptian Propolis: 2. Chemical composition, antiviral and antimicrobial activities of East Nile Delta propolis. Z. Naturforsch. (Germany). 57c(3-4):386-394. 
2. AGRA, R.; RODRIGUES, E.; MARCUCCI, M.; RAMALHO, A.; DOMINGUES, N.; ESFRAIM, W. 2006. Características físico-químicas e atividade antimicrobiana de extratos de própolis da Paraíba, Brasil. Ciência Rural. (Brasil). 36(6):1842-1848.

3. AGRIOS, G.N. 2004. Fitopatología. Ed. Limusa. (México D.C.). $838 p$.

4. AGÜERO, M.B.; SVETAZ, L.; SÁNCHEZ, M.; LUNA, L.; LIMA, B.; LÓPEZ, M.L.; ZACCHINO, S.; PALERMO, J.; WUNDERLIN, D.; FERESIN, G.E.; TAPIA, A. 2011. Argentinean andean propolis associated with the medicinal plant Larrea nitida Cav. (Zygophyllaceae). HPLC-MS and GC-MS characterization and antifungal activity. Food Chem Toxicol. (Netherlands). 49(9):1970-1978.

5. BANKOVA, V.; POPOVA, M.; TRUSHEVA, B. 2014. Propolis volatile compounds: chemical diversity and biological activity: a review. Chem. Cent. J. (United States). 8(28):1-8.

6. BARBARIĆ, M.; MIŠKOVIĆ, K.; BOJIĆ, M.; LONČAR, M.B.; SMOLČIĆ-BUBALO, A.; DEBELJAK, Z.; MEDIĆ-ŠARIĆ, M. 2011. Chemical composition of the ethanolic propolis extracts and its effect on HeLa cells. J. Ethnopharmacol. (Netherlands). 135(3):772-778.

7. CEUGNIEZ, A.; DRIDER, D.; JACQUES, P.; COUCHENEY, F. 2015. Yeast diversity in a traditional French cheese "Tomme d'orchies"reveals infrequent and frequent species with associated benefits. Food Microbiology. (Netherlands). 52(1):177-184.

8. CLINIC AND LABORATORY STANDARDS INSTITUTE -CLSI-. 1997. Reference method for broth dilution antifungal susceptibility testing for yeasts. Approved standard document M27-A. CLSI. 17(9):1-29.

9. CLINIC AND LABORATORY STANDARDS INSTITUTE -CLSI-. 2002. Reference method for broth dilution antifungal susceptibility testing of filamentous fungi. Approved standard M38-A, CLSI. 22(16):1-22.

10. FREIRES, I.A.; QUEIROZ, V.C.P.P.; FURLETTI, V.F.; IKEGAKI, M.; DE ALENCAR, S.M.; DUARTE, M.C.T.; ROSALEN, P.L. 2016. Chemical composition and antifungal potential of Brazilian propolis against Candida spp. J. Mycologie Médicale. (Netherlands). 26(2):122-132.

11. GALINDO, M.P. 1986. Una alternativa de representación simultánea: HJ-biplot. Questió. 10(1):13-23.
12. GÓMEZ-TENORIO, M.A.; ZANÓN, M.J.; DE CARA, M.; LUPIÓN, B.; TELLO, J.C. 2015. Efficacy of dimethyl disulfide (DMDS) against Meloidogyne sp. and three formae speciales of Fusarium oxysporum under controlled conditions. Crop Protection. (Netherlands). 78(1):263-269.

13. GOUMA, M.; GAYÁN, E.; RASOA, J.; CONDÓN, S.; ÁLVAREZ, I. 2015. Inactivation of spoilage yeasts in apple juice by UV-C light and in combination with mild heat. Innovative Food Science and Emerging Technologies. (Netherlands). 32(1):146-155.

14. GRAIKOU, K.; POPOVA, M.; GORTZI, O.; BANKOVA, V.; CHINOU, I. 2016. Characterization and biological evaluation of selected Mediterranean propolis samples. Is it a new type? LWT-Food Science and Technology. (Netherlands). 65(1):261-267.

15. GYAWALI, R.; IBRAHIM, S.A. 2014. Natural products as antimicrobial agents. Food Control. (Netherlands). 46(1):412-429.

16. HERRERA, C.; ALVEAR, M.; BARRIENTOS, L.; MONTENEGRO, G.; SALAZAR, L. 2010. The antifungal effect of six commercial extracts of Chilean propolis on Candida spp. Cien. Inv. Agr. (Chile). 37(1):75-84.

17. JIAO, L.; YUAN, W. 2004. Study on application of propolis in food industry. Food Sci. Technol. (Canada). 1(12):55-57.

18. LUCERA, A.; COSTA, C.; CONTE, A.; DEL NOBILE, M.A. 2012. Food applications of natural antimicrobial compounds. Front. Microbiol. (Switzerland). 3(287):1-13.

19. MENESES, E.A.; DURANGO, D.; GARCÍA, C.M. 2009. Antifungal activity against postharvest fungi by extracts from Colombian propolis. Quím. Nova. (Brasil). 32(8):2011-2017.

20. MELLIOU, E.; STRATIS, E.; CHINOU, I. 2007. Volatile constituents of propolis from various regions of Greece-antimicrobial activity. Food Chem. (Netherlands). 103(2):375-380.

21. ORBERÁ-RATÓN, T.M. 2004. Acción perjudicial de las levaduras sobre los alimentos. Rev. Cubana Salud Pública. (Cuba). 30(3):1-5.

22. ÖZCAN, M.; UNVER, A.; CEYLAN, D.A.; YETIŞIR, R. 2004. Inhibitory effect of pollen and propolis extracts. Nährung. (United States). 48(3):188-194. 
23. ÖZCAN, M. 1999. Antifungal properties of propolis. Grasas y Aceites. (España). 50(5):395-398.

24. PALOMINO, L.; MARTÍNEZ, J.; GARCÍA, C.; GIL, J.; DURANGO, D. 2010. Physicochemical characterization and antimicrobial activity of propolis from municipality of Union (Antioquia, Colombia). Rev. Fac. Nal. Agr. Medellín. (Colombia). 63(1):5373-5383.

25. PAPOTTI, G.; BERTELLI, D.; BORTOLOTTI, L.; PLESSI, M. 2012. Chemical and functional characterization of italian propolis obtained by different harvesting methods. J. Agric. Food. Chem. (United States). 60(11):2852-2862.

26. POP, A.; DRUGAN, T.; GUTLEB, A.C.; LUPU, D.; CHERFAN, J.; LOGHIN, F.; KISS, B. 2016. Individual and combined in vitro (anti)androgenic effects of certain food additives and cosmetic preservatives. Toxicology in Vitro. (Netherlands). 32(1):269-277.

27. QUIROGA, E.N.; SAMPIETRO, D.A.; SOBERON, J.R.; SGARIGLIA, M.A.; VATTUONE, M.A. 2006. Propolis from the northwest of Argentina as a source of antifungal principles. J. Appl. Microbiol. (United States). 101(1):103-110.

28. RIVILLAS-ACEVEDO, L.A.; SORIANO-GARCÍA, M. 2007. Isolation and biochemical characterization of an antifungal peptide from Amaranthus hypochondriacus seeds. J. Agric. Food. Chem. (United States). 55(25):10156-10161.

29. SALATINO, A.; FERNANDES-SILVA, C.C.; RIGHI, A.A.; SALATINO, M.L.F. 2011. Propolis research and the chemistry of plant products. Nat. Prod. Rep. (United Kingdom). 28(5):925-936.

30. SHAHMIHAMMADI, M.; JAVADI, M.; NASSIRI-ASL, M. 2016. An Overview on the Effects of Sodium Benzoa- te as a Preservative in Food Products. Biotech Health Sci. (Iran). In press.

31. SIRIPATRAWAN, U.; VITCHAYAKITTI, W. 2016. Improving functional properties of chitosan films as active food packaging by incorporating with propolis. Food Hydrocolloids. (Netherlands). 61(1):695-702.

32. STEPANOVIĆ, S.; ANTIĆ, N.; DAKIĆ, I.; ŠVABIĆVLAHOVIĆ, M. 2003. In vitro antimicrobial activity of propolis and synergism between propolis and antimicrobial drugs. Microbiol. Res. (Netherlands). 158(4):353-357.

33. SULEMAN, T.; VAN VUUREN, S.; SANDASI, M.; VILJOEN, A.M. 2015. Antimicrobial activity and chemometric modelling of South African propolis. J. of Applied Microbiology. (United States). 119(4):981990.

34. TORETI, V.C.; SATO, H.H.; PASTORE, G.M.; PARK, Y.K. 2013. Recent Progress of propolis for its biological and chemical compositions and its botanical origin. Evid. Based Complement. Alternat. Medi. (Egypt). 2013(1):1-13.

35. TWUMASI, P.; OHENE-MENSAH, G.; MOSES E. 2014. The rot fungus Botryodiplodia theobromae strains cross infect cocoa, mango, banana and yam with significant tissue damage and economic losses. African J. Agr. Res. (Nigeria). 96(6):613-619.

36. YANG, S.Z.; PENG, L.T.; SU, X.J.; CHEN, F.; CHENG, Y.J.; FAN, G.; PAN, S.Y. 2011. Bioassay-guided isolation and identification of antifungal components from propolis against Penicillium italicum. Food. Chem. (Netherlands). 127(1):210-215.

Recibido: Junio 17 de 2016

Aceptado: Septiembre 30 de 2016

\section{Cómo citar:}

Viloria-Barragán, J.; Gil-González, J.; Durango-Restrepo, D.; Marín-Loaiza, J.; Correa-Londoño, G. 2016. Actividad in vitro de extractos etanólicos de propóleos del bajo Cauca antioqueño sobre dos hongos filamentosos y uno levaduriforme. Rev. U.D.C.A Act. \& Div. Cient. 19(2): 333-340. 